\section{Holographic bubble chambers}

from David J. Miller

THE new particles have tantalisingly short lifetimes. Even the $\mathrm{D}^{+}$, the longest-lived charmed particle, lasts for only about $10^{-12}$ seconds - time to travel $300 \mu \mathrm{m}$ at the velocity of light, or a few millimetres with relativistic time dilation. But the $\mathrm{D}^{\circ}$, the $\Delta_{\text {charm }}$, the $\mathrm{F}$ meson, the $\tau$ lepton and the 'beauty' mesons are predicted to travel only some tens of microns before decay. Nuclear emulsions have come back into popularity in the past five years because they gave the only chance of studying such short tracks, and now a new generation of tiny bubble chambers is developing which can resolve bubbles of less than $25 \mu \mathrm{m}$ in diameter. Each technique has shortcomings - the emulsion requires many scanner-years of work to find the interesting events; a bubble chamber with conventional cameras has such a poor depth of field when the resolution drops below $25 \mu \mathrm{m}$ that it becomes impossible to focus all of the beam particles into the sensitive volume.

At the Fermilab workshop* Le Coq (CERN) presented impressive pictures and videotapes that had been reconstructed from holograms made with a $6.5 \mathrm{~cm}$ diameter by $3.5 \mathrm{~cm}$ deep heavy liquid bubble chamber ('BIBC', the Berne Infinitessimal Bubble Chamber). The holographic technique gave clearly resolved images of bubbles only $8 \mu \mathrm{m}$ in diameter over the whole depth of the chamber. This work has inspired a number of other developments. Pless (MIT) presented scaling rules for the change of resolution when Le Coq's 'straightthrough' holographic layout is modified to medium-sized bubble chambers with $\sim 1$ $\mathrm{m}$ diameter. His collaboration is now presenting a proposal to Fermilab for a study of the $\tau$ neutrino in such a chamber, using a neutrino beam from a beam dump. A proposal by a European collaboration for a small holographic hydrogen bubble chamber was discussed by Fisher (Rutherford). This would be a continuation of the work being done with conventional optics in proton and pion beams at CERN. He warned that, although the desired resolution can almost certainly be achieved over a useful volume, and although there are techniques available for detecting, measuring and identifying the secondary particles outside the chamber, one serious problem remains for the exploitation of holographic bubble chambers - a very similar problem to that faced by nuclear emulsions. The new particles are produced very rarely - in less at once set the matter at rest.' than one event in a thousand. To make an emulsion, or a hologram, really useful, an electronic trigger must be devised which might contain the short-lived particle. The tracks from these events must then be extrapolated back into the emulsion or the chamber so that the scanner knows where to look. With a bubble chamber it should be possible, in principle, to pulse a laser and make the hologram only when a good event has been detected, but Fisher warned that no technique yet exists which can separate the rare particles cleanly from the background within the few microseconds that it takes for bubbles to grow to the desired size.

Some people think that an alternative approach might bring good results. Instead of looking for a 'needle in a haystack', using small chambers with fine resolution in proton, pion or photon beams, where the backgrounds are enormous, it is perhaps more sensible to work in a neutrino beam where charmed particles occur in 10 per cent of events. To have a reasonable number of neutrino interactions a large bubble chamber must be used, such as BEBC at CERN or the 15 foot chamber at Fermilab. At present these chambers grow their bubbles to around $600 \mu \mathrm{m}$ in diameter in order to give a clear conventional image from more than $10 \mathrm{~m}^{3}$ of sensitive volume. A few $\mathrm{D}^{+}$tracks have been resolved - the very furthest tail of the length distribution - but many more could be seen if $50 \mu \mathrm{m}$ resolution were possible. Very little work has been done on the possibilities of using holography in the large bubble chambers but no one has demonstrated that it cannot be done. A number of methods were will identify the subsample of events which

suggested: bright field 'Scotchlite' illumination, though work at Imperial College has shown that speckle may be a problem; dark field with Scotchlite, but can sufficiently powerful lasers be found; retrodirecting mirrors, if a suitable reference beam can be set up. Peterson (Hawaii) drew attention to the relevance of these ideas to proposals his collaboration is making for a new chamber at Fermilab. Its primary purpose is to allow secondary particles from neutrino interactions to leave the bubble chamber and be identified, but such a new device could also be designed to incorporate whatever is found to be the most favourable holographic layout.

We were reminded by Lederman, the Fermilab director, that finer resolution means smaller equipment which costs less to build. Fine electronic counters would enable experiments of all kinds to be scaled-down proportionally to the resolution, giving more than proportional reductions in magnet, material and building costs. Such counters were outside the scope of the workshop, but more than one group is convinced that the solution to the 'needle in a haystack' problem will depend on an emulsion or small holographic bubble chamber being followed by electronic counters with a resolution of $20 \mu \mathrm{m}$ which will both improve the trigger and allow very precise predictions to be passed on to the scanners.

The workshop ended with a theorist's checklist of the effects that could be studied at high resolution. Bjorken (Fermilab) stressed that once the new particles can be collected in reasonable numbers they should show as complex and instructive a phenomenology as did the strange particles.

D.J. Miller is a lecturer in the Department of Physics and Astronomy at University College London, at present on sabbatical leave in Fermilab.

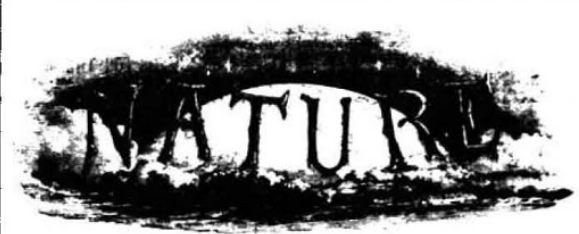

Mr E. T. Sachs sends us some interesting notes from Batavia: - "Within the past month I have been so lucky as to make what I hope is a very interesting if not remarkable discovery. On the Island of Biliton, 200 miles from here, I found a fresh water fish which produces its young living from its mouth. I am quite prepared for the cry of incredulity that will be raised; but I conducted my observations with living fish and closed doors, and what I assert is undeniable: the eggs are hatched in the lower portion of the head of the fish, and are projected out at the mouth and from nowhere else. I have secured several specimens, which I shall send to Dr. Günther, who will of course

100 years ago

\section{A "Natural" Experiment in Polarised Light}

Break off a plate of ice and hold it between the sky and a pool of water. Its reflected image will show the beautiful colours due to polarised light. The incident rays should come from a part of the sky about $90^{\circ}$ from the sun, and reflection should take place at the polarising angle for water, and the plate will probably require adjusting to bring out the maximum effect. Water, vaporous, solid, and liquid, thus furnishes us with polariser, crystal, and analyser. I do not remember to have read any account of this very simple experiment, for which Nature provides all the materials.

Chas. T. Whitmell

9, Beech Grove, Harrogate, January 10

From Nature 33, 20 January, 268, 275, 1881. 